\title{
Glycerol Utilization as Substrate for Ethanol Production in Escherichia coli Recombinant under an Aerobic Condition
}

Wahyu Suradi Pranata ${ }^{1 *}$, Prayoga Suryadarma ${ }^{2}$, Djumali Mangunwidjadja ${ }^{2}$

\author{
${ }^{1}$ Departement of Biotechnology, Postgraduate School of Bogor Agricultural University, Bogor, Indonesia \\ ${ }^{2}$ Departement of Agroindustrial Technology, Faculty of Agriculture Technology, Bogor Agricultural University, Bogor, Indonesia
}

\begin{abstract}
The utilization of glycerol as a substrate for ethanol production in Escherichia coli recombinant harboring ethanologenic gens (PDC and ADHB) under aerobic conditions was investigated. This research was conducted by using $E$. coli BW25113 which compared the growth characteristics on glycerol and glucose as carbon source. E. coli can grow well on both substrates, and it consumed glycerol faster than glucose. On glucose, E. coli occurred overflow metabolism that indicated by high acetate accumulation. Meanwhile, on a substrate of glycerol, the acetate accumulation could be reduced. Using glycerol substrate on $E$. coli increased pyruvate accumulation, comparing with glucose. Therefore, the growth characteristic of glycerol was more efficient. In E. coli $\Delta p t a / p H f d h / p T a d h B$-pdc utilized glycerol substrate was able to accumulate pyruvate as intermediate metabolite for produce $2.18 \mathrm{gL}^{-1}$ ethanol.
\end{abstract}

Keywords: Aerobic condition, Escherichia coli, ethanol, glucose, glycerol

\section{INTRODUCTION}

Energy is an important part of life as most of the human activity requires it. The majority of the energy utilized comes from fossil fuels, especially petroleum, which is non-renewable and potentially collapse to get extinct. Production of ethanol as an eco-friendly renewable fuel is one of the solutions are constantly being developed. The prior generation of ethanol production, fermentation of yeast with substrates derived from starch food products, began to be abandoned because of the food competition and the effect on the economy, then moved to the second generation utilized the biomass substrate of yeast or bacteria fermentation that is more high-technology [1]. The yeast fermentation such as Saccharomyces cerevisiae has the weakness -the slow growth-affecting the outcome. In addition, the bacteria fermentation such as Zymomonas mobilis has a limited spectrum of substrate utilization in sugar, while Escherichia coli has been widely used in engineering microbes for ethanol production because of the well-known performance, rapid growth, and broad spectrum of substra-

${ }^{*}$ Corresponding author:

Wahyu Suradi Pranata

Departement of Biotechnology, Postgraduate School of Bogor Agricultural University

Jalan Raya Darmaga, Bogor, Indonesia 16880

E-mail: calcilcol@gmail.com tes utilization such as glucose and glycerol. Previous studies stated that the role of sugar as a substrate on $E$. coli ethanol fermentation could be replaced by glycerol [2].

Glycerol, also called glycerin, is a trihydrate alcohol compounds with a chemical formula of $\mathrm{C}_{3} \mathrm{H}_{8} \mathrm{O}_{3}$. It is a cheap and plentiful carbon source, easily reduced in metabolism compared with sugar to produce the compounds of reduction such as ethanol, succinic, xylitol, propionic acid, hydrogen, and so on with a higher yield than sugar [3]. Every three moles of biodiesel produced generates one mole of glycerol (about 5-10\% equivalent to the biodiesel weight), for optimizing the potential needs to convert glycerol to be other useful chemicals useful chemicals such as ethanol [4].

Previous studies conducted fermentation in an anaerobic condition, making the slow cell growth and increasing the activity of lactate dehydrogenase (Idh) accumulating lactate using pyruvate substrate. The cultivation of $E$. coli in anaerobic conditions leads to lower the cell growth, resulting in the decline in the expected

\section{How to cite:}

Pranata WS, Suryadarma P, Mangunwidjadja D (2018) Glycerol

Utilization as Substrate for Ethanol Production in Escherichia coli Recombinant under an Aerobic Condition. J. Trop. Life. Science 8 (1): $81-86$ 
product [5]. The anaerobic condition produces the high byproduct, resulted in ineffective ethanol production of E. coli. Thus, the genetic modification is needed [6], and the condition should be converted into aerobic. The ethanologenic gene insertion the genes of pyruvate decarboxylase $(p d c)$ and acetaldehyde dehydrogenase $(a d h B)$ from $Z$. mobilis into $E$. coli is conducted by genetic engineering to increase ethanol production [6]. Another study mentioned the cultivation process under aerobic conditions to produce biomass in large quantities approximately $66 \%$, with the rests of waters, gasses, and organic acids [7]. In addition, the decline of activity under aerobic conditions caused by forming enzyme lactate (Idh) [8] could suppress the lactate accumulation.

The availability of oxygen as a final electron acceptor will consume NADH [9] resulting in the reduction of $\mathrm{NADH}$ for ethanol production. Judging from its metabolic pathway, utilization of glycerol as a substrate is capable to twice increase NADH than glucose [4]. The NADH availability in $E$. coli cultivation also accumulated pyruvate as an intermediate metabolite that utilized to ethanol production [5]. The availability of high oxygen and substrate concentrations leads to the high accumulation of acetate on glucose substrate, called as overflow metabolism [10], which could consequently decrease ethanol production. The use of glycerol as a substrate could reduce the accumulation of acetate [11]. Furthermore, it is expected that ethanologenic E. coli and glycerol substrate could accumulate higher ethanol compared with glucose as acetate, the byproduct of ethanol production can be reduced through the use of glycerol.

Conversion of glycerol to succinic acid is an example of redox balance. Although the pathway for ethanol and succinic is equivalent to overall redox balance, the energy contribution of the ethanologenic pathway is much higher. The energy production of succinic is limited to meet the generation of proton motive force by fumarate reductase ( $f r d$ ) [3]. Therefore, this study would compare glycerol with glucose as a substrate, which is expected to see the potential of glycerol as a substrate for subsequently directed toward ethanol production under aerobic condition.

\section{MATERIALS AND METHODS \\ Bacterial strains}

The strain used in the present study was Escherichia coli BW25113 and its pta deficient (BW25113 4 pta) [12] from the National BioResource Project (National Institute of Genetic (NIG), Mishima, Japan) and used as the host strains. The pHfdh plasmid bearing fdh gene from Mycobacterium vaccae was obtained from a previous study [8]. Plasmid bearing adhB and pdc genes from Zymomonas mobilis constructed by cloning these genes into pTrc99A vector denoted as pTadhB-pdc. This plasmid, pHfdh and/or pTadhB-pdc were transformed into BW25113 and BW25113 $\Delta$ pta strains.

\section{Culture of Escherichia coli strains}

For the cultures, the cells from a glycerol stock were inoculated into $10 \mathrm{~mL}$ of liquid LB medium and incubated at $37^{\circ} \mathrm{C}$ for $12 \mathrm{~h}$ as a pre-culture. The pre-cultured cells were then inoculated into glucose or glycerol enriched LB medium to give an initial optical density at $660 \mathrm{~nm}$ of OD660 $\cong 0.05$. The test medium contained $10 \mathrm{~g}$ polypeptone, $5 \mathrm{~g}$ yeast extract, $10 \mathrm{~g}$ sodium chloride, $220 \mathrm{mM}$ glucose or glycerol per liter of deionized water, antibiotics $(30 \mathrm{mg} / \mathrm{L}$ ampicillin, 15 $\mathrm{mg} / \mathrm{L}$ kanamycin, $34 \mathrm{mg} / \mathrm{L}$ chloramphenicol), together with $0.5 \mathrm{mM}$ isopropyl thiogalactoside (IPTG) for inducing the expression of recombinant protein. The cultures were conducted by adjusting rotary shaking speed $(250 \mathrm{r} / \mathrm{min})$ and volume of medium $50 \mathrm{~mL}$ in 250 $\mathrm{mL}$ baffled conical flasks. The incubation tempe-rature was kept at $37^{\circ} \mathrm{C}$. The initial $\mathrm{pH}$ of the medium was adjusted to 7 and $\mathrm{CaCO} 3(20 \mathrm{~g} / \mathrm{L})$ was added to prevent a $\mathrm{pH}$ drop during culturing. Cell growth was recorded as OD660 after dissolving solid $\mathrm{CaCO} 3$ with dilute $\mathrm{HCl}$, and dry cell weight (DCW) was calculated from DCW= $0.36 \times$ OD660.

\section{Analyses}

Glucose, glycerol, and ethanol concentration in the culture broth was determined by an enzymatic method using F-Kit 716251, F-Kit 148270, F-Kit 176290 (JK International, Tokyo, Japan), respectively, according to the manufacturer's procedure. Acetate and pyruvate concentration was measured by high-performance liquid chromatography (Shimadzu, Kyoto, Japan) with an Aminex HPX-87H column (Bio-Rad, CA, USA), where a mobile phase of $20 \mathrm{mmol} / \mathrm{LH} 2 \mathrm{SO} 4$ flowed at 0.6 $\mathrm{mL} / \mathrm{min}$ and $55^{\circ} \mathrm{C}$, using detection absorbance at 210 $\mathrm{nm}$. Ethanol yields on glycerol basis (Yethanol/glycerol) were determined by the formula:

$$
Y_{\text {ethanol/glycerol }}=\frac{\text { mol } C \text { ethanol }}{\text { mol C glycerol consumption }}
$$

Where the mass of ethanol and mass of glycerol represent the ethanol production or appeared in the culture; and glycerol consumption, respectively. 


\section{RESULTS AND DISCUSSION}

\section{Growth characteristic of glucose and glycerol as carbon} source under aerobic conditions

Escherichia coli was observed in the adaptation phase until the end of the growth phase at 0 until 12 hours, the stationary phase at 12 until 72 hours, found as similar in the general conditions of cell growth on both substrates (Figure 1). The aerobic condition of glycerol substrate administration of adaptation phase until the end of the growth phase could be achieved at 12 hours with the dry cell weight of $3.3 \mathrm{~g} / \mathrm{L}$, literally higher than found by [13] in anaerobic condition ( 84 hours, dry cell weight of $0.31 \mathrm{~g} / \mathrm{L}$ ). Another study with glycerol substrate and aerobic conditions showed the growth of cells reaches the end of the growth phase at the 36 hours [14], 24 hours longer than this study. It might be caused by the different conditions of cultivation. This result indicates the favourable cultivation condition in that might be due to the ability of cells to reach the peak the end of the growth phase quickly, more metabolites produced during this phase. E. coli could growth well, indicating the well-utilize of glycerol and glucose. It is consistent with [15] that the biomass produced by glucose or glycerol did not differ on the condition that oxygen availability acts as a final electron acceptor.

The cell growth is closely related to the consumption of substrate. Glycerol was consumable at 48 hours, while glucose was still available at 72 hours (Figure 1). This result indicates the faster consumption of glycerol than glucose under aerobic condition. It might be indicated by the significantly higher average of reduction rate per carbon $(\mathrm{k})$ of glycerol $\left(\mathrm{C}_{3} \mathrm{H}_{8} \mathrm{O}_{3}: \mathrm{k}=4.67\right)$ than glucose $\left(\mathrm{C}_{6} \mathrm{H}_{12} \mathrm{O}_{6}: \mathrm{k}=4\right)$ [16], showed the more efficient use of glycerol than glucose.

Acetate is a by-product of ethanol production. The accumulation of acetate on glucose is high, but not found on glycerol (Figure 2). This is consistent with [10] in which the high substrate condition and the oxygen availability could drive on the carbon flux direction towards the acetate formation, usually known as overflow metabolism, while the zero-acetate on glycerol is suspected as the simultaneously production and consumption of acetate [11]. These results are favourable for ethanol production by using glycerol because of the capability to suppress the accumulation toward acetate.

Pyruvate, a central metabolite, leads to several metabolites. Pyruvate was accumulated on the glycerol, but not on glucose (Figure 2). This is consistent with [5] which states that pyruvate could be accumulated through the availability of NADH. Glycerol produces twice NADH compared with glucose. The accumulation

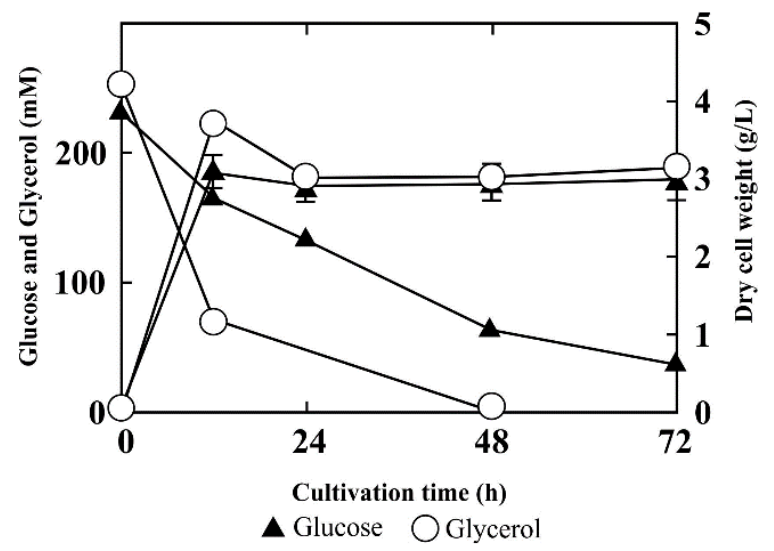

Figure 1. Time profile of DCW, glucose, and glycerol concentration of E. coli BW25113 cultured for 72 hours

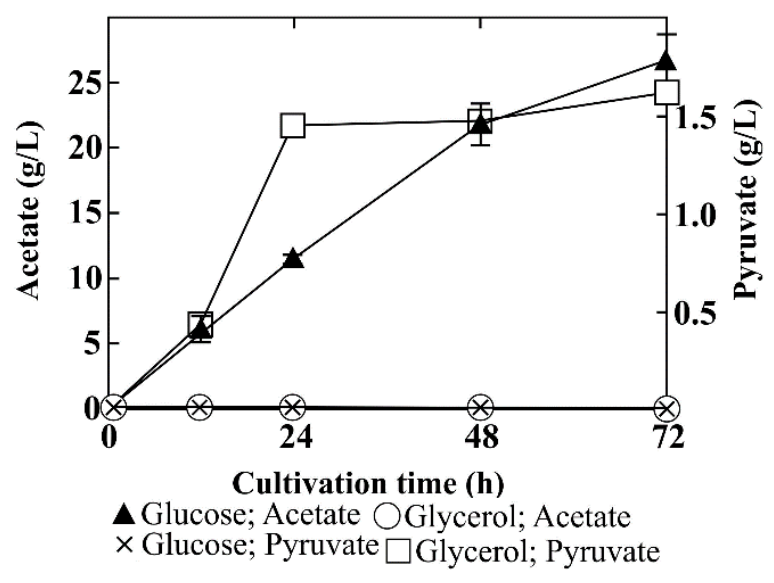

Figure 2. Time profile of acetate and pyruvate concentration of E. coli BW25113 cultured for 72 hours

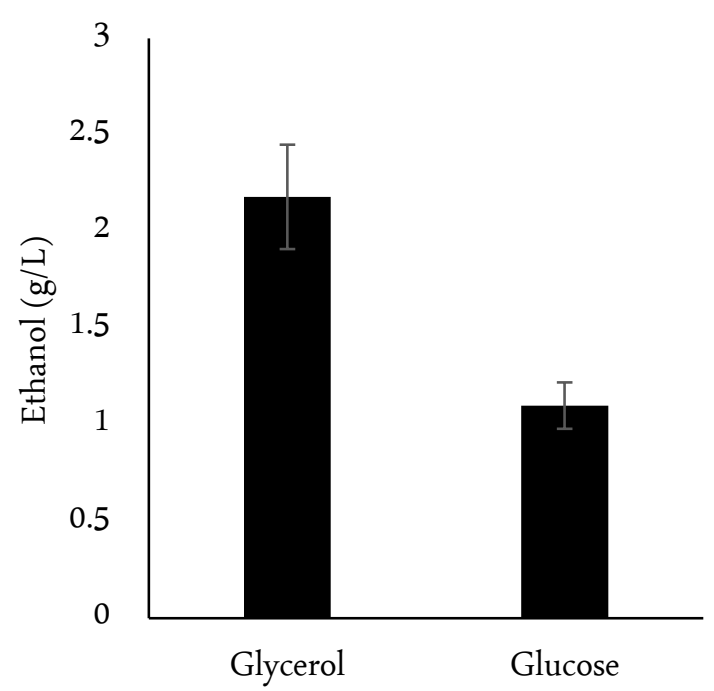

Figure 3. Ethanol accumulation in cultures of the BW25113 $\Delta \mathrm{pta} / \mathrm{pHfdh} / \mathrm{pTadhB}-\mathrm{pdc} E$. coli strain at different substrates. The cells were cultured for $\mathrm{t}=72$ hours. Note: $\left({ }^{*}\right)$ with addition $4 \mathrm{~g} / \mathrm{L}$ formate and no addition IPTG 
Table 1. The utilization of pure and crude glycerol for bioethanol production by different strains of $E$. coli under aerobic condition

\begin{tabular}{lccc}
\hline Strain & Initial glycerol concentration $(\mathrm{g} / \mathrm{L})$ & Ethanol yields $(\mathrm{mol} / \mathrm{mol})$ & Source \\
\hline Pure glycerol & & & \\
E. coli & & & This study \\
$\quad$ BW25113 $\Delta_{p t a / p H f d h / p T a d h B-p d c}$ & 20.45 & 0.16 & {$[19]$} \\
TCS099/pLO1297 & 40 & & \\
Crude glycerol & & & {$[14]$} \\
E. coli & & 0.006 & {$[14]$} \\
BL21_WT & 30 & 0.04 & {$[14]$} \\
BL21_pARD33 & 30 & 0.06 & \\
BL21 pARRD33-pBH2 & 30 & & \\
\end{tabular}

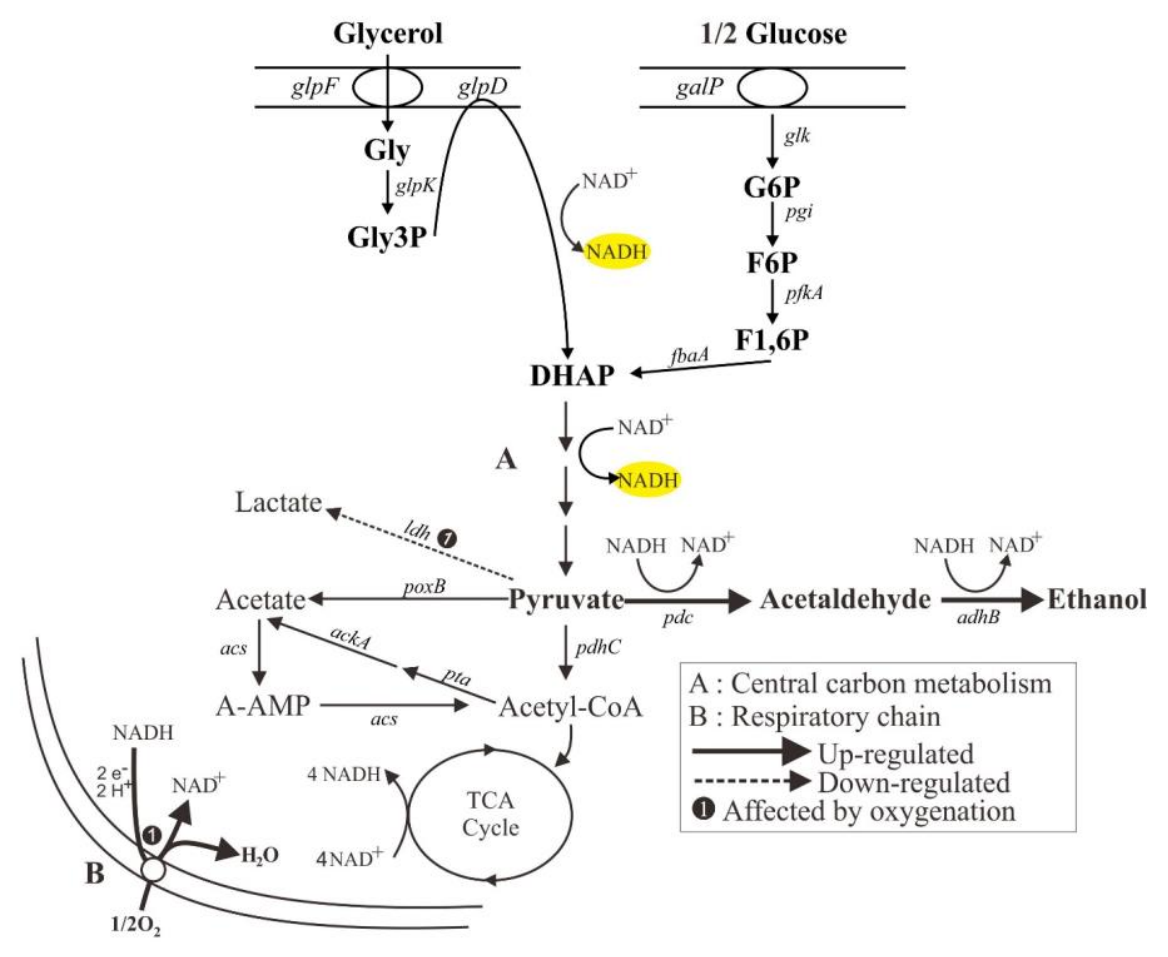

Figure 4. Summary of central carbon metabolism in E. coli ethanologenic recombinant under an aerobic condition

of pyruvate as the intermediate compound is very advantageous for ethanol production through $p d c$ gene insertion that converts pyruvate to acetaldehyde, then $a d h B$ genes that alter acetaldehyde into ethanol, both genes were derived from $Z$. mobilis because of its low Km value.

\section{Ethanol production in Escherichia coli ethanologenic}

Various substrates are potential for ethanol production, including glycerol and glucose. E. coli BW25113 $\Delta \mathrm{pta} / \mathrm{pHfdh} / \mathrm{pTadhB}$-pdc with glycerol as a substrate was able to accumulate ethanol at $2.18 \mathrm{~g} / \mathrm{L}$ higher than glucose that accumulates $1.1 \mathrm{~g} / \mathrm{L}$ (Figure 3). The accumulation of ethanol from pyruvate acetaldehyde-ethanol pathway by pdc and adhB requires the co-substrate
$\mathrm{NADH}$. This result proves that glycerol can provide $\mathrm{NADH}$, in line with [4], two times higher than glucose.

The comparison of ethanol indicates the highest yield resulted by glycerol (Table 1 ). The yield on glycerol is 0.16 , literally higher than the highest achievement of [14] by genetic engineering using E. coli BL21_pARR $\mathrm{D} 33-\mathrm{pBH} 2$ that inserts alrD and aldH genes. The alrD as an oxidative catalyst of glycerol into D-glyceraldehyde to produce NADPH, aldH as a catalyst of D-glyceraldehyde into D-glycerate by regenerating $\mathrm{NADH}$ from $\mathrm{NAD}+$. Then the accumulation of D-glycerate towards the glycolytic pathway to be pyruvate and acetyl-CoA under aerobic condition, directed to the availability of $\mathrm{NADH}$ that be seen from its metabolic pathway engineering strategies. It indicates the more efficient genetic 
engineering strategy for ethanol production.

\section{Metabolism of ethanol production on glycerol and glu- cose substrates}

The metabolism of ethanologenic E. coli with the utilization of glycerol and glucose as a substrate under aerobic conditions is shown in Figure 4. Glycerol, like other small molecules, can pass through the cytoplasmic membrane via passive diffusion. Diffusion is achieved by facilitated by integral membrane proteins (glpF) [17]. The intracellular glycerol is phosphorylated to glycerol-3phosphate (Gly3P) by glycerol kinase (glpK), herein after dehydrogenase to dihydroxyacetone phosphate (DHAP) regenerating $\mathrm{NADH}$ from $\mathrm{NAD}+$ by glpD, then DHAP is catabolized in the pentose-phosphate pathway. Meanwhile, glucose passes through the cytoplasmic membrane glucose facilitated by integral membrane proteins (galP). Glucose kinase (glk) phosphorylate glucose to glucose-6-phosphate (G6P), then converted into frukose-6-phosphate (F6P). F6P is phosphorylated to fructose-1,6-phosphate $(\mathrm{F} 1,6 \mathrm{P})$, then converted to DHAP. The difference in metabolism between two substrates to DHAP is the existence of NADH regeneration only in glycerol substrate. The growth of $E$. coli cells in glycerol substrate under aerobic conditions could form DHAP in the metabolism central I which metabolites it plays a role in gluconeogenesis and glycolysis [18]. NADH was generated from DHAP towards pyruvate on both substrates. Furthermore, pyruvate, as an intermediate metabolite, led to the other metabolites.

The insertion of pdc and adhB genes from $Z$. mobilis with plasmid pTrc directs pyruvate toward ethanol to acetaldehyde with $\mathrm{NADH}$ as the co-substrate. Besides, pyruvate led to lactate by ldh, but the lactate accumulation could be suppressed in aerobic condition [5]. Acetate, the byproduct, that be competing with the ethanol production was produced directly from pyruvate by pyruvate oxidase (poxB) and also from pyruvate to acetylCoA, acetyl-CoA to acetate by the pospho-trans-acetylase (pta) and acetate kinase (ackA). Accumulation of acetate on glycerol substrate can be suppressed, allegedly because acetic simultaneously produced and consumed (Figure 4), possible for acetate to be converted to acetyl phosphate (A-AMP), then converted to acetyl-CoA. Both of these processes were acted by the acetyl-CoA synthetase (acs), while it was unregulated on glucose substrate. It regenerated $\mathrm{NADH}$ from acetyl-CoA to citric. Citric acid cycle plays a major role in the growth as it generates energy. Respiration processed in the outer membrane employed oxygen $\left(\mathrm{O}_{2}\right)$ as the final electron acceptor that is converted to dihydrogen monoxide
$\left(\mathrm{H}_{2} \mathrm{O}\right)$ by converting NADH to NAD+ (Figure 4$)$.

\section{CONCLUSION}

Using glycerol substrate on recombinant E. coli increased pyruvate accumulation, suppressed the accumulation acetate as a by-product. Therefore, the using of glycerol as carbon source enhanced ethanol accumulation under aerobic conditions, comparing with glucose.

\section{ACKNOWLEDGMENT}

We wish to thank National Bioresource Project (National Institute of Genetics (NIG), Mishima, Japan) on E. coli cells strain BW25113 and Department of Chemical Science and Engineering, Osaka University, Japan on pHfdh and pTadhB-pdc plasmids.

\section{REFERENCES}

1. International Energy Agency (2008) From 1st to 2nd-generation biofuel technologies. An overview of current Industry and RD and D Activities. Paris Cedex, OECD/IEA.

2. Ito T, Nakashimada Y, Senba K et al. (2005) Hydrogen and ethanol production from glycerol containing wastes discharged after biodiesel manufacturing process. Journal of Bioscience and Bioengineering 100 (3): $260-265$.

3. Dharmadi Y, Murarka A, Gonzalez R (2006) Anaerobic fermentation of glycerol by Escherichia coli: a new platform for metabolic engineering. Biotechnology and Bioengineerin 94 (5): $821-829$.

4. Yazdani SS, Gonzalez R (2007) Anaerobic fermentation of glycerol: a path to economic viability for the biofuels industry. Current Opinion in Biotechnology 18 (3): 213 - 219.

5. Suryadarma P, Ojima Y, Fukuda Y et al. (2012) The rpoS deficiency suppresses acetate accumulation in glucose-enriched culture of Escherichia coli under an aerobic condition. Frontiers of Chemical Science and Engineering 6 (2): $152-$ 157. doi: 10.1007/s11705-012-1287-0.

6. Ingram LO, Conway T, Clark DP et al. (1987) Genetic engineering of ethanol production in Escherichia coli. Applied and Environmental Microbiology 53 (10): 2420 - 2425.

7. Sutapa IDA (1999) Lumpur aktif: Alternatif pengolah limbah cair. Jurnal Studi Pembangunan, Kemasyarakatan dan Lingkungan 1 (3): 25 - 38.

8. Ojima Y, Kawase D, Nishioka M, Taya M (2009) Functionally undefined gene, yggE, alleviates oxidative stress generated by monoamine oxidase in recombinant Escherichia coli. Biotechnology Letters 31: 139 - 145 .

9. Causey $\mathrm{TB}$, Shanmugam KT, Yomano LP, Ingram LO (2004) Engineering Escherichia coli for efficient conversion of glucose to pyruvate. Proceedings of the National Academy of Sciences of the United States of America 101 (8): 2235 - 
2240.

10. Vemuri GN, Altman E, Sangurdekar DP et al. (2006) Overflow metabolism in Escherichia coli during steady-state growth: Transcriptional regulation and effect of the redox ratio. Applied and Environmental Microbiology 72 (5): 3653 -3661 .

11. Martinez-Gomez K, Flores N, Castaneda HM et al. (2012) New insights into Escherichia coli metabolism: carbon scavenging, acetate metabolism and carbon recycling responses during growth on glycerol. Microbial Cell Factories 11: 1 21. doi: 10.1186/1475-2859-11-46.

12. Baba T, Ara T, Hasegawa M et al. (2006) Construction of Escherichia coli K-12 in-frame, single gene knockout mutants: the keio collection. Molecular Systems Biology 2 (1): 1 - 11. doi: 10.1038/msb4100050.

13. Murarka A, Dharmadi Y, Yazdani SS, Gonzalez R (2008) Fermentative utilization of glycerol by Escherichia coli and its implications for the production of fuels and chemicals. Applied and Environmental Microbiology 74 (4): 1124 1135. doi: 10.1128/AEM.02192-07.

14. Shah P, Chiu F, Lan JC (2014) Aerobic utilization of crude glycerol by recombinant Escherichia coli for simultaneous production of poly 3-hydroxybutyrate and bioethanol. Journal of Bioscience and Bioengineering 117 (3): 343 - 350. doi: 10.1016/j.jbiosc.2013.08.018.

15. Durnin G, Clomburg J, Yeates Z et al. (2008) Understanding and harnessing the microaerobic metabolism of glycerol in Escherichia coli. Biotechnology and Bioengineering 103: 148-161. doi: 10.1002/bit.22246

16. Nielsen J, Villadsen J, Liden G (2003) Bioreaction engineering principles. New York, Kluwer Academic/Plenum Publishers.

17. Darbon E, Ito K, Huang HS et al. (1999) Glycerol transport and phosphoenolpyruvate-dependent enzyme I- and Hprcatalyzed phosphorylation of glycerol kinase in Thermus flavus. Microbiology 145: 3205 - 3212. doi: 10.1099/002212 87-145-11-3205.

18. Frankel DG (1996) Glycolysis. In: Neidhardt FC (eds) Escherichia coli and Salmonella tiphymurium: Cellular and molecular biology. 2nd edition. Washington DC, ASM Press.

19. Trinh CT, Srienc F (2009) Metabolic engineering of Escherichia coli for efficient conversion of crude glycerol to ethanol. Applied and Environmental Microbiology 75 (21): 6696 - 6705. doi: 10.1128/AEM.00670-09. 